\title{
Short-Time Impact of Soil Amendments with Medicago Plant Materials on Soil Nematofauna
}

 \\ 1 Institute of Parasitology, Slovak Academy of Sciences, Hlinkova 3, 04001 Košice, Slovakia \\ 2 Department of Pesticides' Control and Phytopharmacy, Benaki Phytopathological Institute, 8 S. Delta Str., \\ 14561 Athens, Greece; nntali@agro.auth.gr \\ 3 Institute for Sustainable Plant Protection-CNR, 70126 Bari, Italy; trifone.daddabbo@ipsp.cnr.it \\ * Correspondence: renco@saske.sk; Tel.: +30-2310-807572
}

check for updates

Citation: Renčo, M.; Ntalli, N.;

D'Addabbo, T. Short-Time Impact of Soil Amendments with Medicago Plant Materials on Soil Nematofauna. Plants 2021, 10, 145. https://doi.org/ $10.3390 /$ plants10010145

Received: 23 December 2020 Accepted: 10 January 2021

Published: 12 January 2021

Publisher's Note: MDPI stays neutral with regard to jurisdictional clai$\mathrm{ms}$ in published maps and institutional affiliations.

Copyright: $\odot 2021$ by the authors. Licensee MDPI, Basel, Switzerland. This article is an open access article distributed under the terms and conditions of the Creative Commons Attribution (CC BY) license (https:// creativecommons.org/licenses/by/ $4.0 /)$.

\begin{abstract}
Soil amendments with plant materials from Medicago species are widely acknowledged for a suppressive effect on plant-parasitic nematodes but their impact on beneficial components of soil nematofauna is still unknown. A study on potted tomato was carried out to investigate the short-time effects on the overall nematofauna of dry biomasses from six different Medicago species, i.e., M. sativa, M. heyniana, M. hybrida, M. lupulina, M. murex and M. truncatula, incorporated to natural soil at 10, 20, or $40 \mathrm{~g} \mathrm{~kg}^{-1}$ soil rates. All amendments resulted in a significant decrease of the total nematofauna biomass, whereas total abundance was significantly reduced only by $M$. heyniana, M. hybrida, and M. lupulina biomasses. Almost all the Medicago amendments significantly reduced the relative abundance of plant-parasites and root fungal feeders. All amendments significantly increased the abundance of bacterivores, whereas fungivores significantly increased only in soil amended with M. heyniana, M. lupulina and M. sativa plant materials. Mesorhabditis and Rhabditis were the most abundant genera of bacterivores, whereas Aphelenchoides and Aphelenchus prevailed among the fungivores. Predators were poorly influenced by all the tested Medicago biomasses, whereas the abundance of omnivores was negatively affected by M. heyniana and M. lupulina. Values of the Maturity Index and Sum Maturity Index were reduced by treatments with M. heyniana, M. hybrida, M. lupulina and M. sativa plant materials, whereas most of the tested amendments decreased values of the Channel Index while increasing those of the Enrichment Index. Enrichment and bacterivore footprints raised following soil addition with Medicago biomasses, whereas composite and fungivore footprints were significantly reduced. According to their overall positive effects on soil nematofauna, amendments with Medicago plant materials or their formulated derivatives could represent an additional tool for a sustainable management of plant-parasitic nematodes.
\end{abstract}

Keywords: Medicago species; plant biomasses; soil amendments; nematode community soil food web descriptors

\section{Introduction}

The economic impact of plant-parasitic nematodes on agricultural crops imposes the use of control measures aiming to reduce their soil population densities under damage thresholds [1]. The EU (European Union) restrictions of the use of plant protection products (Reg. EC 889/2008; 128/2009; 1185/2009) [2] has given a strong impulse to research on safer control alternatives to synthetic nematicides, including the use of soil organic amendments based on agroindustrial wastes, crop residual biomasses, or green manures [3,4]. The suppressiveness of these materials to phytonematodes could involve several concurrent or alternative mechanisms, such as the stimulation of soil nematode- antagonistic microflora or the release of nematicidal compounds [5,6], but is still not clearly elucidated.

Soil incorporation of fresh or dry biomasses from several plants has been frequently proven to have a suppressive effect on various plant-parasitic nematodes, such as root-knot species (Meloidogyne spp.) cyst-forming nematodes (Globodera spp., Heterodera spp.) or 
ectoparasite species (Pratylenchus spp., Xiphinema index) [7-13]. In particular, soil treatments with dry biomasses from Medicago species repeatedly resulted in a strong reduction of root-knot and cyst nematode infestations both in pot and field experiments [14-17], mainly due to the high content of preformed nematotoxic saponins [18] and to the release of toxic ammoniacal compounds by tissue degradation into the soil $[19,20]$.

In addition to plant parasitism, components of soil nematofauna can show several more feeding behaviors, according to which they are usually classified into five basic trophic groups, i.e., bacterivores, fungivores, omnivores, predators, and plant parasites [21]. All these trophic groups give a relevant contribution to soil food web functions, driving important soil ecosystem processes (microbial spread, organic matter decomposition, nitrogen mineralization, and more) [22-27].

Abundance, ubiquitous distribution, and a close relationship with other soil food web components make nematodes able to promptly respond to the environmental changes and, therefore, represent affordable bioindicators of any perturbation occurring in soil [23,24,27-30]. According to nematode trophic groups' response to variations of soil conditions, a number of ecological indices have been developed, such as the maturity (MI), enrichment (EI), structure (SI), and channel (CI) index as well as the metabolic footprints [24,31,32]. These indices allow the monitoring of soil health conditions following any ecosystem disturbance, including agronomical practices such as organic amendments' soil incorporation [33-35].

Components of soil nematofauna were found to be modified after soil amendments with plant biomasses. Such effects on the abundance and presence of particular trophic groups however depend on the amount and composition of the amendment raw materials. Populations of bacterivore and fungivore trophic groups were found to increase after soil amendments with different raw or composted residues of cotton (Gossypium hirsutum L.) and rye (Secale cereale L.) mixed to vetch (Vicia sativa L.) [36], or maize (Zea mays L.), Texas panicum (Panicum texanum Buckley R. Webster) and velvet bean (Mucuna pruriens L. DC.) [37]. A bacterial decomposition pathway was also observed following soil incorporation with green biomasses of Brassicaceae plants such as yellow mustard (Sinapis alba L.) and radish (Raphanus sativus L.) [38], whereas a greater fungal-based food web occurred in soil amended with rapeseed (Brassica napus L.) and rye, constantly with no changes in omnivore and predator abundance [39]. There is no previous study on the impact of soil amendments with Medicago plant materials on the non-parasitic, beneficial components of soil nematode community. Therefore, a study was carried out to assess the short-time effects of soil amendments with biomasses from the same six different Medicago species as previously tested on the root-knot nematode $M$. incognita, i.e., M. sativa, M. heyniana, M. hybrida, M. lupulina, M. murex, and M. truncatula [17], on the overall nematofauna of natural tomato-planted soil.

\section{Results}

\subsection{Nematode Abundance and Biomass}

Nematode abundance was differently affected by the six Medicago plant biomasses' incorporation (Figure 1A). Compared to the non-amended control, a significantly lower abundance was observed in soil amended with M. heyniana, M. hybrida, and M. lupulina. In contrast, the incorporation of the biomasses from M. murex, M. sativa, and M. truncatula resulted in no significant impact on overall nematode abundance. No significant variation of total nematode individuals occurred among the different amendment rates, except for the highest rate of M. lupulina. Adversely to abundance, all amendments with the Medicago plant materials resulted in a significant decrease of the total nematode biomass, irrespective of the applied incorporation rate (Figure 1B). 



Figure 1. Total nematode abundance (A) and biomass (B) in soil amended with 10, 20, or $40 \mathrm{~g} \mathrm{~kg}^{-1}$ soil rates of dry plant biomass of six Medicago plant species.

\subsection{Nematode Trophic Groups}

Nematofauna of non-amended soil was prevalently constituted by bacterivores (41.4\%), plant parasitic nematodes $(33.7 \%)$, and fungivores $(14.6 \%)$, with a low presence of rootfungal feeders $(5.4 \%)$, omnivores (3.9\%), and predators (Figure 2). Relative abundance of bacterivores was significantly increased by the incorporation of biomasses from all the Medicago species except $M$. truncatula. Adversely, only M. truncatula amendments resulted in a significant increase of herbivores, i.e., plant parasites, as significantly reduced by all the other Medicago biomasses. Relative abundance of fungivores significantly raised in soil amended with M. heyniana, M. lupulina, and Megicago sativa plant materials, but was not statistically affected by the other three Medicago species. Only M. heyniana and M. lupulina amendments negatively affected the abundance of omnivores, whereas all six species biomasses significantly reduced the incidence of root fungal feeders and poorly influenced the presence of predators. All the recorded effects were not significantly related to the amendment rates. 



RF

Figure 2. Relative abundance of the different nematode trophic groups in soil amended with 10, 20, or $40 \mathrm{~g} \mathrm{~kg}^{-1} \mathrm{soil} \mathrm{rates}$ of dry plant biomass from six different Medicago species. $\mathrm{BA}=$ bacterivores; $\mathrm{FU}=$ fungivores; $\mathrm{OM}=$ omnivores; $\mathrm{PP}=$ plant parasites; PR = predators; and RF = root fungal feeders.

\subsection{Nematode Genera}

A total of 31 nematode genera were identified in non-treated soil, whereas this number ranged from a minimum of 22 (treatment with $10 \mathrm{~g} \mathrm{~kg}^{-1}$ soil of M. lupulina biomass) to 30 (M. sativa at $10 \mathrm{~g} \mathrm{~kg}^{-1}$ soil) in the amended soil (Table S1). Bacterivore genera were the most numerous (15), followed by omnivores (5), fungivores, as well as plant parasites and predators (3), whereas only two genera of root-fungal feeders were identified. Within bacterivores, Acrobeloides, Cephalobus, Plectus, Mesorhabditis, and Rhabditis were the most abundant genera, whereas Aphelenchoides and Aphelenchus prevailed among the fungivores.

All the tested amendments increased the abundance of only two nematode genera, the bacterivore Rhabditis and the fungivore Aphelenchus. Among plant parasites, the population of genus Meloidogyne was significantly reduced, whereas that of Geocenamus strongly increased in soil amended with M. lupulina, M. murex, and M. truncatula biomasses, as giving account of the highest nematode abundance recorded for the $10 \mathrm{~g} \mathrm{~kg}^{-1}$ soil treatment with the $M$. truncatula biomass.

\subsection{Soil Food Web Indices}

Compared to control, the Maturity Index (MI) and Sum Maturity Index ( $\Sigma \mathrm{MI})$ were significantly higher in soil treated with M. heyniana, M. hybrida, M. lupulina, and M. sativa plant material (Table 1). 
Table 1. Soil food web descriptors in soil amended with dry biomasses from six Medicago species.

\begin{tabular}{|c|c|c|c|c|c|c|c|c|c|c|c|c|}
\hline $\begin{array}{c}\text { Rate } \\
\text { (g kg }{ }^{-1} \text { soil) }\end{array}$ & MI & & PPI & & MI 2-5 & & $\sum \mathbf{M I}$ & & CI & & EI & \\
\hline \multicolumn{13}{|c|}{ M. heyniana } \\
\hline 0 & $2.09 \pm 0.05$ & $\mathrm{a}$ & $2.96 \pm 0.05$ & $\mathrm{a}$ & $2.21 \pm 0.07$ & a & $2.48 \pm 0.09$ & a & $48.9 \pm 15.6$ & a & $43.9 \pm 8.46$ & $\mathrm{a}$ \\
\hline 10 & $1.47 \pm 0.11$ & $\mathrm{~b}$ & $3.00 \pm 0.00$ & $\mathrm{a}$ & $2.17 \pm 0.14$ & a & $1.62 \pm 0.11$ & $\mathrm{~b}$ & $9.6 \pm 4.7$ & $\mathrm{~b}$ & $86.8 \pm 5.6$ & $\mathrm{~b}$ \\
\hline 20 & $1.66 \pm 0.5$ & $b$ & $2.96 \pm 0.02$ & $\mathrm{a}$ & $2.07 \pm 0.03$ & $\mathrm{a}$ & $1.82 \pm 0.12$ & $\mathrm{~b}$ & $18.5 \pm 4.5$ & $\mathrm{~b}$ & $75.4 \pm 4.1$ & $\mathrm{~b}$ \\
\hline 40 & $1.59 \pm 0.12$ & $\mathrm{~b}$ & $3.00 \pm 0.00$ & $\mathrm{a}$ & $\begin{array}{r}2.12 \pm 0.04 \\
\text { M. hybrida }\end{array}$ & $\mathrm{a}$ & $1.64 \pm 0.18$ & $\mathrm{~b}$ & $14.9 \pm 5.0$ & $\mathrm{~b}$ & $81.2 \pm 5.1$ & $\mathrm{~b}$ \\
\hline 0 & $2.09 \pm 0.05$ & $\mathrm{a}$ & $2.96 \pm 0.05$ & $\mathrm{a}$ & $2.21 \pm 0.07$ & a & $2.48 \pm 0.09$ & $\mathrm{a}$ & $48.9 \pm 15.6$ & a & $43.9 \pm 8.46$ & $\mathrm{a}$ \\
\hline 10 & $1.53 \pm 0.28$ & $\mathrm{~b}$ & $2.98 \pm 0.02$ & $\mathrm{a}$ & $2.42 \pm 0.09$ & $\mathrm{~b}$ & $1.76 \pm 0.23$ & $\mathrm{~b}$ & $7.6 \pm 3.0$ & $\mathrm{~b}$ & $90.2 \pm 3.9$ & $\mathrm{~b}$ \\
\hline 20 & $1.71 \pm 0.22$ & $\mathrm{a}$ & $2.98 \pm 0.02$ & $\mathrm{a}$ & $2.29 \pm 0.03$ & a & $1.89 \pm 0.22$ & $\mathrm{~b}$ & $17.4 \pm 8.5$ & $\mathrm{~b}$ & $80.3 \pm 8.6$ & $\mathrm{~b}$ \\
\hline 40 & $1.69 \pm 0.15$ & $\mathrm{~b}$ & $2.99 \pm 0.02$ & $\mathrm{a}$ & $\begin{array}{l}2.11 \pm 0.07 \\
\text { M. lupulina }\end{array}$ & $\mathrm{a}$ & $1.77 \pm 0.17$ & $\mathrm{~b}$ & $14.5 \pm 5.2$ & $\mathrm{~b}$ & $73.2 \pm 10.4$ & $\mathrm{~b}$ \\
\hline 0 & $2.09 \pm 0.05$ & $\mathrm{a}$ & $2.96 \pm 0.05$ & $\mathrm{a}$ & $2.21 \pm 0.07$ & $\mathrm{a}$ & $2.48 \pm 0.09$ & $\mathrm{a}$ & $48.9 \pm 15.6$ & $\mathrm{a}$ & $43.9 \pm 8.46$ & $\mathrm{a}$ \\
\hline 10 & $1.84 \pm 0.01$ & $\mathrm{a}$ & $3.00 \pm 0.00$ & $\mathrm{a}$ & $2.05 \pm 0.03$ & $\mathrm{a}$ & $1.91 \pm 0.05$ & $\mathrm{~b}$ & $31.9 \pm 4.3$ & $\mathrm{a}$ & $60.6 \pm 2.1$ & $\mathrm{~b}$ \\
\hline 20 & $1.79 \pm 0.05$ & $\mathrm{~b}$ & $2.88 \pm 0.04$ & $\mathrm{a}$ & $2.08 \pm 0.04$ & $\mathrm{a}$ & $1.82 \pm 0.05$ & $\mathrm{~b}$ & $27.4 \pm 7.9$ & $\mathrm{~b}$ & $66.8 \pm 5.3$ & $\mathrm{~b}$ \\
\hline 40 & $1.79 \pm 0.16$ & $\mathrm{~b}$ & $3.00 \pm 0.00$ & $\mathrm{a}$ & $\begin{array}{r}2.12 \pm 0.07 \\
\text { M. murex }\end{array}$ & a & $1.82 \pm 0.15$ & $\mathrm{~b}$ & $31.1 \pm 13.3$ & $\mathrm{a}$ & $67.8 \pm 12.2$ & $\mathrm{~b}$ \\
\hline 0 & $2.09 \pm 0.05$ & $\mathrm{a}$ & $2.96 \pm 0.05$ & $\mathrm{a}$ & $2.21 \pm 0.07$ & a & $2.48 \pm 0.09$ & $\mathrm{a}$ & $48.9 \pm 15.6$ & a & $43.9 \pm 8.46$ & $\mathrm{a}$ \\
\hline 10 & $2.08 \pm 0.05$ & $\mathrm{a}$ & $3.00 \pm 0.00$ & $\mathrm{a}$ & $2.27 \pm 0.09$ & a & $2.36 \pm 0.12$ & $\mathrm{a}$ & $32.8 \pm 13.7$ & $\mathrm{a}$ & $39.9 \pm 9.7$ & $\mathrm{a}$ \\
\hline 20 & $2.03 \pm 0.07$ & $\mathrm{a}$ & $2.95 \pm 0.07$ & $\mathrm{a}$ & $2.12 \pm 0.07$ & a & $2.24 \pm 0.07$ & $\mathrm{a}$ & $48.8 \pm 9.2$ & $\mathrm{a}$ & $41.4 \pm 8.2$ & $\mathrm{a}$ \\
\hline 40 & $2.09 \pm 0.06$ & $\mathrm{a}$ & $2.96 \pm 0.02$ & $\mathrm{a}$ & $\begin{array}{r}2.17 \pm 0.07 \\
\text { M. sativa }\end{array}$ & a & $2.32 \pm 0.04$ & $\mathrm{a}$ & $40.8 \pm 14.1$ & $\mathrm{a}$ & $34.9 \pm 9.8$ & $\mathrm{a}$ \\
\hline 0 & $2.09 \pm 0.05$ & $\mathrm{a}$ & $2.96 \pm 0.05$ & $\mathrm{a}$ & $2.21 \pm 0.07$ & a & $2.48 \pm 0.09$ & $\mathrm{a}$ & $48.9 \pm 15.6$ & $\mathrm{a}$ & $43.9 \pm 8.46$ & $\mathrm{a}$ \\
\hline 10 & $1.96 \pm 0.09$ & $\mathrm{a}$ & $2.98 \pm 0.02$ & $\mathrm{a}$ & $2.13 \pm 0.04$ & a & $2.01 \pm 0.15$ & $\mathrm{~b}$ & $36.5 \pm 9.2$ & $\mathrm{a}$ & $52.5 \pm 9.7$ & $\mathrm{a}$ \\
\hline 20 & $1.69 \pm 0.06$ & $\mathrm{~b}$ & $2.96 \pm 0.02$ & $\mathrm{a}$ & $2.09 \pm 0.04$ & a & $1.73 \pm 0.06$ & $b$ & $17.2 \pm 4.2$ & $\mathrm{~b}$ & $74.4 \pm 4.7$ & $\mathrm{~b}$ \\
\hline 40 & $1.86 \pm 0.14$ & $\mathrm{a}$ & $2.98 \pm 0.04$ & $\mathrm{a}$ & $\begin{array}{l}2.14 \pm 0.09 \\
\text { M. truncatula }\end{array}$ & a & $1.91 \pm 0.14$ & $b$ & $27.9 \pm 12.6$ & $b$ & $64.9 \pm 6.6$ & $\mathrm{~b}$ \\
\hline 0 & $2.09 \pm 0.05$ & $\mathrm{a}$ & $2.96 \pm 0.05$ & $\mathrm{a}$ & $2.21 \pm 0.07$ & a & $2.48 \pm 0.09$ & $\mathrm{a}$ & $48.9 \pm 15.6$ & $\mathrm{a}$ & $43.9 \pm 8.46$ & $\mathrm{a}$ \\
\hline 10 & $1.94 \pm 0.39$ & $\mathrm{a}$ & $3.00 \pm 0.00$ & $\mathrm{a}$ & $2.36 \pm 0.30$ & a & $2.41 \pm 0.21$ & $\mathrm{a}$ & $19.2 \pm 11.9$ & $\mathrm{~b}$ & $72.5 \pm 8.3$ & $\mathrm{~b}$ \\
\hline 20 & $1.91 \pm 0.15$ & $\mathrm{a}$ & $2.99 \pm 0.02$ & $\mathrm{a}$ & $2.29 \pm 0.12$ & a & $2.42 \pm 0.21$ & $\mathrm{a}$ & $22.0 \pm 5.8$ & $\mathrm{~b}$ & $70.5 \pm 6.1$ & $\mathrm{~b}$ \\
\hline 40 & $1.71 \pm 0.13$ & $\mathrm{~b}$ & $3.00 \pm 0.00$ & $\mathrm{a}$ & $2.25 \pm 0.09$ & a & $2.33 \pm 0.11$ & $\mathrm{a}$ & $15.5 \pm 5.1$ & $\mathrm{~b}$ & $78.8 \pm 8.4$ & $\mathrm{~b}$ \\
\hline
\end{tabular}

Each value is a mean of five replications. Data marked with the same letter in each column are not statistically different to untreated control according Least Significant Difference Test $(p=0.05)$. MI = Maturity index; PPI = Plant parasitic index; MI2-5 = Maturity index for colonizers-persisters group 2-5; $\Sigma \mathrm{MI}=$ Sum Maturity index; $\mathrm{CI}=$ Channel index; EI—Enrichment index.

Adversely, the values of Plant Parasitic Index (PPI) and MI2-5 were not significantly affected by any soil amendment with Medicago plant biomass. The Channel index (CI) significantly decreased in pots treated with M. heyniana, M. hybrida, M. sativa, and M. truncatula, while the Enrichment index (EI) resulted in an increase by the amendments with plant materials from all the Medicago species except for M. murex.

Significant differences among the non-treated control and amended soil were also found for the values of nematode metabolic footprints (Table 2). In particular, soil amendments with Medicago plant materials always resulted in a significant dose-related decrease of composite and fungivore footprints and, adversely, in significantly higher values of enrichment and bacterivore footprints. 
Table 2. Nematode metabolic footprints (means \pm SE) after soil treatment with dry biomass from six Medicago species.

\begin{tabular}{|c|c|c|c|c|c|c|c|c|c|c|c|c|c|c|}
\hline $\begin{array}{c}\text { Rate } \\
\left(\mathrm{g} \mathrm{kg}^{-1} \text { soil) }\right.\end{array}$ & Cfoot & & Efoot & & Hfoot & & Ffoot & & Bfoot & & Pfoot & & \multicolumn{2}{|l|}{ Ofoot } \\
\hline \multicolumn{15}{|c|}{ M. heyniana } \\
\hline 0 & $\begin{array}{c}2027.6 \pm \\
158.9\end{array}$ & $\mathrm{a}$ & $\begin{array}{c}78.5 \pm \\
23.3\end{array}$ & a & $\begin{array}{c}1807.2 \pm \\
92.4\end{array}$ & a & $\begin{array}{c}18.8 \pm \\
5.7\end{array}$ & a & $\begin{array}{c}124.2 \pm \\
37.9\end{array}$ & a & $\begin{array}{c}11.6 \pm \\
3.6\end{array}$ & $\mathrm{a}$ & $\begin{array}{c}65.9 \pm \\
12.7\end{array}$ & $\mathrm{a}$ \\
\hline 10 & $\begin{array}{c}573.5 \pm \\
58.8\end{array}$ & $\mathrm{~b}$ & $\begin{array}{c}303.8 \pm \\
52.4\end{array}$ & $\mathrm{~b}$ & $\begin{array}{c}237.5 \pm \\
31.8\end{array}$ & $\mathrm{~b}$ & $20.2 \pm 2.4$ & a & $\begin{array}{c}302.9 \pm \\
69.7\end{array}$ & $\mathrm{~b}$ & $3.6 \pm 2.1$ & $\mathrm{a}$ & $9.5 \pm 4.6$ & $\mathrm{~b}$ \\
\hline 20 & $\begin{array}{c}354.2 \pm \\
18.8\end{array}$ & $\mathrm{~b}$ & $\begin{array}{c}177.3 \pm \\
28.8\end{array}$ & $\mathrm{~b}$ & $\begin{array}{c}127.5 \pm \\
53.8\end{array}$ & $\mathrm{~b}$ & $\begin{array}{c}24.9 \pm \\
8.1\end{array}$ & a & $\begin{array}{c}175.9 \pm \\
55.8\end{array}$ & a & $1.8 \pm 0.6$ & $\mathrm{a}$ & $\begin{array}{c}23.9 \pm \\
14.2\end{array}$ & $\mathrm{~b}$ \\
\hline 40 & $\begin{array}{c}492.8 \pm \\
44.4\end{array}$ & $\mathrm{~b}$ & $\begin{array}{c}317.5 \pm \\
44.7\end{array}$ & $\mathrm{~b}$ & $\begin{array}{c}109.0 \pm \\
13.7\end{array}$ & $\mathrm{~b}$ & $\begin{array}{c}29.9 \pm \\
6.4\end{array}$ & $\mathrm{~b}$ & $\begin{array}{c}307.7 \pm \\
94.4\end{array}$ & $b$ & $3.2 \pm 2.0$ & $\mathrm{a}$ & $\begin{array}{c}43.6 \pm \\
17.8\end{array}$ & $a b$ \\
\hline \multicolumn{15}{|c|}{ M. hybrida } \\
\hline 0 & $\begin{array}{c}2027.6 \pm \\
158.9\end{array}$ & $\mathrm{a}$ & $\begin{array}{c}78.5 \pm \\
23.3\end{array}$ & a & $\begin{array}{c}1807.2 \pm \\
92.4\end{array}$ & a & $\begin{array}{c}18.8 \pm \\
5.7\end{array}$ & a & $\begin{array}{c}124.2 \pm \\
37.9\end{array}$ & a & $\begin{array}{c}11.6 \pm \\
3.5\end{array}$ & $\mathrm{a}$ & $\begin{array}{c}65.9 \pm 1 \\
2.7\end{array}$ & $\mathrm{a}$ \\
\hline 10 & $\begin{array}{c}648.5 \pm \\
88.7\end{array}$ & $\mathrm{~b}$ & $\begin{array}{c}193.8 \pm \\
61.1\end{array}$ & $\mathrm{~b}$ & $\begin{array}{c}391.1 \pm \\
45.6\end{array}$ & $\mathrm{~b}$ & $\begin{array}{c}11.2 \pm \\
2.1\end{array}$ & $\mathrm{~b}$ & $\begin{array}{c}189.9 \pm \\
38.88\end{array}$ & $\mathrm{~b}$ & $3.5 \pm 1.8$ & $\mathrm{a}$ & $\begin{array}{c}52.8 \pm \\
23.1\end{array}$ & $\mathrm{a}$ \\
\hline 20 & $\begin{array}{c}558.5 \pm \\
55.9\end{array}$ & $\mathrm{~b}$ & $\begin{array}{c}186.8 \pm \\
56.8\end{array}$ & $\mathrm{~b}$ & $\begin{array}{c}297.5 \pm \\
77.2\end{array}$ & $\mathrm{~b}$ & $\begin{array}{c}20.2 \pm \\
3.9\end{array}$ & a & $\begin{array}{c}183.0 \pm \\
67.36\end{array}$ & $\mathrm{~b}$ & $9.4 \pm 2.6$ & $\mathrm{~b}$ & $\begin{array}{c}48.5 \pm \\
15.2\end{array}$ & $\mathrm{a}$ \\
\hline 40 & $\begin{array}{c}488.8 \pm \\
35.4\end{array}$ & $\mathrm{~b}$ & $\begin{array}{c}207.6 \pm \\
33.8\end{array}$ & $\mathrm{~b}$ & $\begin{array}{c}232.9 \pm \\
55.7\end{array}$ & $\mathrm{~b}$ & $\begin{array}{c}20.4 \pm \\
4.0\end{array}$ & a & $\begin{array}{c}226.8 \pm \\
77.1\end{array}$ & $\mathrm{~b}$ & $3.0 \pm 1.2$ & $\mathrm{a}$ & $\begin{array}{c}14.3 \pm \\
3.2\end{array}$ & $\mathrm{~b}$ \\
\hline \multicolumn{15}{|c|}{ M. lupulina } \\
\hline 0 & $\begin{array}{c}2027.6 \pm \\
158.9\end{array}$ & $\mathrm{a}$ & $\begin{array}{c}78.5 \pm \\
23.3\end{array}$ & a & $\begin{array}{c}1807.2 \pm \\
92.4\end{array}$ & a & $\begin{array}{c}18.8 \pm \\
5.7\end{array}$ & a & $\begin{array}{c}124.2 \pm \\
37.9\end{array}$ & a & $\begin{array}{c}11.6 \pm \\
3.5\end{array}$ & $\mathrm{a}$ & $\begin{array}{c}65.9 \pm \\
12.7\end{array}$ & $\mathrm{a}$ \\
\hline 10 & $\begin{array}{c}506.3 \pm \\
69.9\end{array}$ & $\mathrm{~b}$ & $\begin{array}{c}350.2 \pm \\
88.2\end{array}$ & $\mathrm{~b}$ & $\begin{array}{c}80.8 \pm \\
12.8\end{array}$ & $\mathrm{~b}$ & $\begin{array}{c}36.5 \pm \\
11.3\end{array}$ & $\mathrm{~b}$ & $\begin{array}{c}368.9 \pm \\
83.2\end{array}$ & $\mathrm{~b}$ & $5.1 \pm 3.0$ & $\mathrm{~b}$ & $\begin{array}{c}15.0 \pm \\
5.9\end{array}$ & $\mathrm{~b}$ \\
\hline 20 & $\begin{array}{c}514.5 \pm \\
60.3\end{array}$ & $\mathrm{~b}$ & $\begin{array}{c}392.8 \pm \\
69.4\end{array}$ & $\mathrm{~b}$ & $\begin{array}{c}60.2 \pm \\
10.7\end{array}$ & $\mathrm{~b}$ & $\begin{array}{c}32.6 \pm \\
10.8\end{array}$ & $\mathrm{~b}$ & $\begin{array}{c}403.7 \pm \\
61.2\end{array}$ & $\mathrm{~b}$ & $6.5 \pm 1.5$ & $\mathrm{~b}$ & $\begin{array}{c}11.8 \pm \\
6.8\end{array}$ & $\mathrm{~b}$ \\
\hline 40 & $\begin{array}{c}879.7 \pm \\
55.6\end{array}$ & $\mathrm{~b}$ & $\begin{array}{c}745.3 \pm \\
101.2\end{array}$ & $\mathrm{~b}$ & $\begin{array}{c}62.3 \pm \\
30.8\end{array}$ & $\mathrm{~b}$ & $\begin{array}{c}50.1 \pm \\
8.3\end{array}$ & $\mathrm{~b}$ & $\begin{array}{c}732.4 \pm \\
115.2\end{array}$ & $\mathrm{~b}$ & $6.9 \pm 2.5$ & $\mathrm{~b}$ & $\begin{array}{c}28.3 \pm \\
12.4\end{array}$ & $\mathrm{~b}$ \\
\hline \multicolumn{15}{|c|}{ M. murex } \\
\hline 0 & $\begin{array}{c}2027.6 \pm \\
158.9\end{array}$ & $\mathrm{a}$ & $\begin{array}{c}78.5 \pm \\
23.3\end{array}$ & a & $\begin{array}{c}1807.2 \pm \\
92.4\end{array}$ & $\mathrm{a}$ & $\begin{array}{c}18.8 \pm \\
5.7\end{array}$ & $\mathrm{a}$ & $\begin{array}{c}124.2 \pm \\
37.9\end{array}$ & $\mathrm{a}$ & $\begin{array}{c}11.6 \pm \\
3.6\end{array}$ & $\mathrm{a}$ & $\begin{array}{c}65.9 \pm \\
12.7\end{array}$ & $\mathrm{a}$ \\
\hline 10 & $\begin{array}{c}698.8 \pm \\
64.5\end{array}$ & $\mathrm{~b}$ & $\begin{array}{c}115.8 \pm \\
23.9\end{array}$ & $\mathrm{~b}$ & $\begin{array}{c}418.7 \pm \\
115.9\end{array}$ & $\mathrm{~b}$ & $\begin{array}{c}13.3 \pm \\
5.4\end{array}$ & $\mathrm{a}$ & $\begin{array}{c}197.8 \pm \\
52.3\end{array}$ & $\mathrm{~b}$ & $\begin{array}{c}10.2 \pm \\
4.2\end{array}$ & $\mathrm{~b}$ & $\begin{array}{c}58.6 \pm \\
10.2\end{array}$ & $\mathrm{a}$ \\
\hline 20 & $\begin{array}{c}681.4 \pm \\
47.7\end{array}$ & $\mathrm{~b}$ & $\begin{array}{c}154.5 \pm \\
30.6\end{array}$ & $\mathrm{~b}$ & $\begin{array}{c}330.3 \pm \\
86.1\end{array}$ & $\mathrm{~b}$ & $\begin{array}{c}34.8 \pm \\
12.8\end{array}$ & $\mathrm{~b}$ & $\begin{array}{c}215.0 \pm \\
36.9\end{array}$ & $\mathrm{~b}$ & $3.5 \pm 1.2$ & $\mathrm{a}$ & $\begin{array}{c}97.9 \pm \\
18.2\end{array}$ & $\mathrm{~b}$ \\
\hline 40 & $\begin{array}{c}573.8 \pm \\
88.2\end{array}$ & $\mathrm{~b}$ & $\begin{array}{c}122.1 \pm \\
54.7\end{array}$ & $\mathrm{~b}$ & $\begin{array}{c}240.3 \pm \\
52.4\end{array}$ & $\mathrm{~b}$ & $\begin{array}{c}16.4 \pm \\
3.9\end{array}$ & $\mathrm{a}$ & $\begin{array}{c}204.5 \pm \\
54.5\end{array}$ & $\mathrm{~b}$ & $\begin{array}{c}11.5 \pm \\
3.7\end{array}$ & $\mathrm{~b}$ & $\begin{array}{c}101.1 \pm \\
31.3\end{array}$ & $\mathrm{~b}$ \\
\hline \multicolumn{15}{|c|}{ M. sativa } \\
\hline 0 & $\begin{array}{c}2027.6 \pm \\
158.9\end{array}$ & $\mathrm{a}$ & $\begin{array}{c}78.5 \pm \\
23.3\end{array}$ & a & $\begin{array}{c}1807.2 \pm \\
92.4\end{array}$ & $a$ & $\begin{array}{c}18.8 \pm \\
5.7\end{array}$ & $\mathrm{a}$ & $\begin{array}{c}124.2 \pm \\
37.9\end{array}$ & $\mathrm{a}$ & $11.6 \pm 3.6$ & $\mathrm{a}$ & $\begin{array}{c}65.9 \pm \\
12.7\end{array}$ & $\mathrm{a}$ \\
\hline 10 & $\begin{array}{c}635.8 \pm \\
66.2\end{array}$ & $\mathrm{~b}$ & $\begin{array}{c}374.7 \pm \\
55.7\end{array}$ & $\mathrm{~b}$ & $\begin{array}{c}107.5 \pm \\
27.2\end{array}$ & $\mathrm{~b}$ & $\begin{array}{c}35.8 \pm \\
6.7\end{array}$ & $\mathrm{~b}$ & $\begin{array}{c}422.1 \pm \\
69.4\end{array}$ & $\mathrm{~b}$ & $2.3 \pm 0.6$ & $\mathrm{~b}$ & $\begin{array}{c}67.2 \pm \\
20.2\end{array}$ & $\mathrm{a}$ \\
\hline 20 & $\begin{array}{c}1152.8 \pm \\
114.8\end{array}$ & $\mathrm{~b}$ & $\begin{array}{c}972.4 \pm \\
83.7\end{array}$ & $\mathrm{~b}$ & $\begin{array}{c}93.8 \pm \\
15.5\end{array}$ & $\mathrm{~b}$ & $\begin{array}{c}35.0 \pm \\
8.2\end{array}$ & $\mathrm{~b}$ & $\begin{array}{c}998.0 \pm \\
155.5\end{array}$ & $\mathrm{~b}$ & $1.3 \pm 0.2$ & $\mathrm{~b}$ & $\begin{array}{c}24.7 \pm \\
6.7\end{array}$ & $\mathrm{~b}$ \\
\hline 40 & $\begin{array}{c}518.7 \pm \\
99.1\end{array}$ & $\mathrm{~b}$ & $\begin{array}{c}348.0 \pm \\
69.3\end{array}$ & $\mathrm{~b}$ & $\begin{array}{c}73.9 \pm \\
29.6\end{array}$ & $\mathrm{~b}$ & $\begin{array}{c}23.4 \pm \\
6.9\end{array}$ & $\mathrm{a}$ & $\begin{array}{c}377.9 \pm \\
57.2\end{array}$ & $\mathrm{~b}$ & $2.8 \pm 1.0$ & $\mathrm{~b}$ & $\begin{array}{c}41.0 \pm \\
11.1\end{array}$ & $\mathrm{~b}$ \\
\hline \multicolumn{15}{|c|}{ M. truncatula } \\
\hline 0 & $\begin{array}{c}2027.6 \pm \\
158.9\end{array}$ & $\mathrm{a}$ & $\begin{array}{c}78.5 \pm \\
23.3\end{array}$ & a & $\begin{array}{c}1807.2 \pm \\
92.4\end{array}$ & a & $\begin{array}{c}18.8 \pm \\
5.7\end{array}$ & $\mathrm{a}$ & $\begin{array}{c}124.2 \pm \\
37.9\end{array}$ & $\mathrm{a}$ & $\begin{array}{c}11.6 \pm \\
3.6\end{array}$ & $\mathrm{a}$ & $\begin{array}{c}65.9 \pm \\
12.7\end{array}$ & $\mathrm{a}$ \\
\hline 10 & $\begin{array}{c}1034.5 \pm \\
42.6\end{array}$ & $\mathrm{~b}$ & $\begin{array}{c}394.9 \pm \\
51.1\end{array}$ & $\mathrm{~b}$ & $\begin{array}{c}496.2 \pm \\
60.2\end{array}$ & $\mathrm{~b}$ & $\begin{array}{c}23.9 \pm \\
3.7\end{array}$ & $\mathrm{a}$ & $\begin{array}{l}417.9 \\
\pm 36.8\end{array}$ & $\mathrm{~b}$ & $\begin{array}{c}11.2 \pm \\
3.1\end{array}$ & $\mathrm{a}$ & $\begin{array}{c}85.5 \pm \\
21.1\end{array}$ & $\mathrm{a}$ \\
\hline 20 & $\begin{array}{c}910.1 \pm \\
63.8\end{array}$ & $\mathrm{~b}$ & $\begin{array}{c}304.3 \pm \\
65.2\end{array}$ & $\mathrm{~b}$ & $\begin{array}{c}541.8 \pm \\
98.3\end{array}$ & $\mathrm{~b}$ & $\begin{array}{c}16.5 \pm \\
5.6\end{array}$ & $\mathrm{a}$ & $\begin{array}{c}318.3 \pm \\
48.3\end{array}$ & $\mathrm{~b}$ & $3.2 \pm 1.2$ & $\mathrm{~b}$ & $\begin{array}{c}31.6 \pm \\
8.3\end{array}$ & $\mathrm{~b}$ \\
\hline 40 & $\begin{array}{c}1040.8 \pm \\
86.4\end{array}$ & $\mathrm{~b}$ & $\begin{array}{c}389.1 \pm \\
12.7\end{array}$ & $\mathrm{~b}$ & $\begin{array}{c}564.0 \pm \\
61.3\end{array}$ & $\mathrm{~b}$ & $\begin{array}{c}14.9 \pm \\
5.5\end{array}$ & $\mathrm{a}$ & $\begin{array}{c}394.4 \pm \\
63.7\end{array}$ & $\mathrm{~b}$ & $5.5 \pm 1.7$ & $\mathrm{~b}$ & $\begin{array}{c}62.1 \pm \\
5.9\end{array}$ & $\mathrm{a}$ \\
\hline
\end{tabular}

Each value is a mean of five replications. Data marked with the same letter in each column are not statistically different to untreated control according Least Significant Difference Test $(p=0.05)$. Cfoot $=$ Composite footprint; Efoot $=$ Enrichment footprint; Hfoot $=$ Herbivore footprint; Ffoot = Fungivore footprint; Bfoot = Bacterivore footprint; Pfoot = Predator footprint; Ofoot = Omnivore footprint. 


\section{Discussion}

Nematodes play a key role within the soil food web due to their strict relationship with soil microorganisms and their functions $[24,31,39]$. In consideration of this key position, the analysis of soil nematofauna has been largely acknowledged as an effective tool for the assessment of soil health and quality status following chemical, physical, and agricultural perturbations, also including soil organic amendments [33,35,40].

In this study, soil incorporation with the biomasses of the six Medicago plants caused an overall decrease of nematofauna abundance and biomass. Contrastingly, most literature studies generally reported an increase of total nematode abundance following soil amendments with various organic materials [12,40-44]. However, the observed decrease of nematofauna abundance and biomass was mainly related to the strong suppression of plant parasites, while beneficial nematofauna components such as bacterivores, fungivores, and omnivores were almost generally increased. This is the first report of the effects of soil amendments with Medicago plant materials on the whole soil nematofauna, as the previous studies were limited to the assessment of the impact of various Medicago plant biomasses on selected plant-parasitic nematode species [14-17].

The increase of bacterivore abundance observed in our study is in good agreement with literature data, which reported a larger presence of bacterivore and fungivore nematofauna after soil amendments with biomasses from other Leguminosae plants, such as vetch or velvet bean [36,37]. A bacterial decomposition pathway was also observed in soil incorporated with green biomasses of Brassicaceae plants as white mustard and radish [12], whereas the prevalence of a fungal-based food web was observed in soil amended with rapeseed (Brassica napus L.) and rye [38]. The large feeding substrate provided to bacterial and fungal populations by the incorporated Medicago biomasses can reasonably explain the shift of soil nematofauna towards the bacterivore and fungivore trophic groups, as also stated by previous literature studies [29,45-48]. An increased populations of bacterivorous and fungivorous nematodes was assessed also after soil amendments with other Leguminosae plants, such as vetch or velvetbean [36,37]. Genera Rhabditis, Mesorhabditis, Acrobeloides, Plectus, and Cephalobus were the most abundant among bacterivores, whereas the species of Aphelenchus and, at a lesser extent, Aphelenchoides prevailed among the fungivores. A prevalence of Rhabditidae and Cephalobidae bacterivores was also observed in the studies of Bulluck et al. [36] and Forge et al. [49], as well as Porazinska et al. [50] describing a dramatic increase of Rhabditidae bacterivores following to compost amendments in orchard soils. Moreover, McSorley and Frederick [37] reported a prevalence of genera Aphelenchoides and Aphelenchus within the fungivore nematofauna also following soil amendments with velvet bean biomass. Analogously, our findings of an increased abundance of omnivores, as well as of limited or nil effects on predators, are also corroborated by the the results from previous studies in fields amended with various organic materials $[12,47,48]$. As indicated for bacterivores, shifts of omnivore and predator populations should be related to the alteration of their food sources more than to a direct toxic effect of Medicago plant materials $[40,43]$. Finally, the strong suppressing effect showed by the biomasses from $M$. heyniana, M. hybrida, M. murex, and M. sativa on plant-parasitic nematofauna fits well the results from our previous comparative study on $M$. incognita on tomato [17], though in the absence of the direct relationship of suppressive effects with the amendment rates. However, the generation time of a majority of free-living nematode genera is still unknown, as it is very difficult or almost impossible to determine, and all the characteristics of feeding groups or c-p gropus are assumptions based on ecological and/or nematological studies $[21,24,28,31,32]$. Consequently, we do not know if the lifecycle of the found nematode genera correspond to our experimental time, i.e., they all were able to reproduce in that time frame or if that could have affected the final results. Therefore, our considerations are necessarily limited to the short-time impact of the tested amendments on the detected nematode genera, while further time-extended studies should be needed to assess the long period population dynamics. Nematode community indices were developed as synthetic indicators of the status of soil food web as well as of the soil 
environment [24]. Values of Maturity Indices (MI, MI2-5 and $\sum$ MI) are generally reduced by any shifts of soil nematofauna towards more adaptable nematode species, i.e., species with a high reproductive activity, short life cycle, and high tolerance to changes of soil conditions (e.g., Rhabditidae, Panagrolaimidae), such as those occurring after organic amendments. In good agreement with data from our current study, lower values of maturity indices in soil amended with various organic matrices than in non-treated soil were also documented by previous literature studies [36,42,46].

The Enrichment Index (EI) is based on the expected responsiveness of the opportunistic guilds (bacterivorous nematodes with c-p value equal to one) to organic resource enrichment. Therefore, EI describes whether the soil food web is nutrient enriched (high EI) or depleted (low EI) [24]. On the other hand, values of Channel Index (CI) indicate the predominant decomposition channel in the soil food web. A high CI (over 50\%) indicates a higher proportion of fungal decomposition and reflect the high relative abundance of c-p fungal feeders (Aphelenchoididae and Aphelenchidae) and the corresponding low abundance of c-p1 bacterial feeders (Rhabditidae and Panagrolaimidae), whereas a low CI (under 50\%) suggests a bacterial decomposition channel. Literature studies generally described an EI increase and a CI decrease in soils amended with organic materials compared to non-amended control, due to a predominant bacterial decomposition channel [12,51-53]. Therefore, the low values of CI and high values of EI, as well as the significant decrease of the values of all maturity indices, recorded in soil amended with most of Medicago plant biomasses indicate that also these materials can nutritionally enrich the soil environment and thus support the bacterial decomposition channel, despite the increased number of fungivores in amended soils.

A calculation of the metabolic footprint of different nematode trophic groups enables a functional quantification of biomass, metabolic activity, and magnitude of carbon and energy flow occurring in the soil food web through their respective channels [32,54]. In this study, values of metabolic footprints indicate that most $C$ flow in soil amended with Medicago plants biomasses occurred through the bacterial channel. However, there is also a relevant $C$ flow through the fungal channel in soil amended with $M$. lupulina and $M$. sativa biomasses, as indicated by the significantly higher fungivore footprint and density.

\section{Materials and Methods}

\subsection{Experimental Design}

Natural sandy soil (64.4\% sand,18.7\% silt, 16.9\% clay, $0.8 \% \mathrm{OM}$, pH 7.5) was collected from a field located at Castellaneta (province of Taranto, Apulia region). The soil was thoroughly mixed and treated with 10,20 , or $40 \mathrm{~g} \mathrm{~kg}^{-1}$ soil rates of the same batches of dry green biomasses of $M$. heyniana, M. hybrida, M. lupulina, M. murex, M. sativa, and $M$. truncatula used in the previous study by D'Addabbo et al. [17]. The amended soil was then poured into $1.5 \mathrm{~L}$ clay pots, providing five replicates for each treatment and including non-treated soil as a control. As in D'Addabbo et al. [17], a one-month-old tomato seedling (cv Tomito) was transplanted in each pot two weeks after the soil incorporation with the Medicago plant biomasses. Plants were maintained in a glasshouse $\left(25 \pm 2{ }^{\circ} \mathrm{C}\right.$ constant temperature) for a two month period. The experiments were repeated twice.

\subsection{Nematode Extraction and Identification}

A composite $100 \mathrm{~mL}$ soil sample was collected from each pot and soaked for $30 \mathrm{~min}$ in $1 \mathrm{~L}$ of tap water. Samples were then processed by a combination of Cobb's sieving and decanting method [55] and a modified Baermann technique [56]. The extracted nematodes were at first examined under a stereomicroscope and then fixed with a hot 99:1 solution of $4 \%$ formaldehyde: pure glycerol. Fixed nematodes were microscopically (Nikon Eclipse 90i light microscope) identified to a genera level according to original species descriptions and taxonomic keys [57-60]. Nematode abundance in each sample was expressed as the number of individuals per $100 \mathrm{~g}$ of dry soil after gravimetrically measuring soil moisture content by oven drying $\left(105^{\circ} \mathrm{C}\right.$ for $\left.24 \mathrm{~h}\right) 100 \mathrm{~g}$ of soil to constant weight. 


\subsection{Nematode Identification and Classification}

Nematode genera were partitioned to 6 trophic groups, i.e., bacterivores (B), fungivores (F), root-fungal feeders (facultative plant parasites) (RFF), obligatory plant parasites $(\mathrm{PP})$, predators $(\mathrm{P})$, and omnivores $(\mathrm{O})$, according to Yeates et al. [21] and Wasilewska [61] and following adjustments and supplementations of Sieriebriennikov et al. [62]. Genera were also assigned to the colonizer-persister (c-p) 1-5 scale based on their $r$ and k characteristics, according to Bongers and Bongers [28] and Bongers [31]. In particular, C-p1 taxa consist of "r-strategists", with short generation times, small eggs, and high fecundity, whereas c-p5 taxa consist of "k-strategists", with long generation times, large bodies, low fecundity, and high sensitivity to disturbance [24]. The total number of genera, total nematode abundance, relative abundance of nematodes per trophic group, and nematode biomass were also determined.

\subsection{Ecological and Functional Indices}

Changes in the nematode communities were also evaluated by calculating different ecological and functional indices and metabolic footprints. Ecological indices as maturity index MI [31], sum maturity index $\sum$ MI [63], maturity index MI2-5 [63] for non-parasitic nematodes and plant parasitic index PPI [31], functional indices enrichment index EI and channel index CI (24), nematode biomass by Andrassy's formula [64], and metabolic footprints were calculated using the NINJA online software [62].

\subsection{Statistical Analysis}

Data from the two runs of the experiment were pooled as no significant experiment $x$ treatment interactions were found [65]. The pooled data were arcsin-transformed as to homogenize error variances and then subjected to one-way analysis of variance, comparing means by the Least Significant Difference Test at $p \leq 0.05$ [65]. All statistical analyses were performed by the PlotIT 3.2 (Scientific Programming Enterprises, Haslett, MI, USA) software.

\section{Conclusions}

This study indicated that soil amendments with biomasses from Medicago plants could not only suppress the plant-parasitic nematodes but also increase the beneficial components of the soil nematode community, such as the bacterivore and fungivore species. Medicago materials seem to be particularly suitable to nematicidal soil amendments, as operating a biological soil disinfestation from plant parasites due to their high content of bioactive saponins and the release of biocidal ammoniacal nitrogen [16], while acting at the same time as a food source for beneficial trophic groups. More generally, the association of reduced plant-parasitic nematode infestations and related soilborne fungal diseases to an improved soil quality could result in better crop growth and crop yield, as also confirmed by previous experiments in the field [15]. According to these overall effects, soil amendments with Medicago plant materials or their formulated derivatives could be suggested as an additional tool for a sustainable control phytonematode.

Supplementary Materials: The following are available online at https:/ / www.mdpi.com/2223-774 7/10/1/145/s1, Table S1: Total abundance (individuals $500 \mathrm{~g}^{-1}$ soil) ${ }^{1}$ of nematode genera in soil amended with 10, 20 or $40 \mathrm{~g} \mathrm{~kg}^{-1}$ soil rates of dry biomasses from the six tested Medicago species.

Author Contributions: Conceptualization, M.R. and T.D.; investigation, M.R. and T.D.; data analysis, M.R.; writing-original draft preparation, M.R., T.D. and N.N.; writing-review and editing, N.N. and T.D. All authors have read and agreed to the published version of the manuscript.

Funding: This research received no external funding.

Acknowledgments: The authors are grateful to project Vega no. 2/0018/20.

Conflicts of Interest: The authors declare no conflict of interest. 


\section{References}

1. Nicol, J.M.; Turner, S.J.; Coyne, D.L.; Nijs, L.; Hockland, S.; Maafi, Z.T. Current nematode threats to world agriculture. In Genomics and Molecular Genetics of Plant-Nematode Interactions; Jones, J., Gheysen, G., Fenoll, C., Eds.; Springer: Dordrecht, The Netherlands, 2011; pp. 21-43.

2. Möhring, N.; Dalhaus, T.; Enjolras, G.; Finger, R. Crop insurance and pesticide use in European agriculture. Agric. Syst. 2020, 184, 102902. [CrossRef]

3. Akhtar, M.; Malik, A. Roles of organic soil amendments and soil organisms in the biological control of plant-parasitic nematodes: A review. Biores. Technol. 2000, 74, 35-47. [CrossRef]

4. Renčo, M. Organic amendments of soil as useful tools of plant parasitic nematodes control. Helminthologia 2013, 50, 3-14. [CrossRef]

5. Bailey, K.L.; Lazarovits, G. Suppressing soil-borne diseases with residue management and organic amendments. Soil Till. Res. 2003, 72, 169-180. [CrossRef]

6. Oka, Y. Mechanisms of nematode suppression by organic soil amendments-a review. Appl. Soil Ecol. 2010, 44, 101-115. [CrossRef]

7. Kimpinski, J.; Arsenault, W.J.; Gallant, C.E.; Sanderson, J.B. The effect of marigolds (Tagetes spp.) and other cover crops on Pratylenchus penetrans and on following potato crops. J. Nematol. 2000, 32, 531-536.

8. Aballay, E.; Sepúlveda, R.; Insunza, V. Evaluation of five nematode-antagonistic plants used as green manure to control Xiphinema index Thorne et Allen on Vitis vinifera L. Nematropica 2004, 34, 45-52.

9. Tsay, T.T.; Wu, S.T.; Lin, Y.Y. Evaluation of Asteraceae plants for control of Meloidogyne incognita. J. Nematol. 2004, 36, 36-41.

10. Wang, K.H.; McSorley, R.; Gallaher, R.N. Effect of Crotalaria juncea amendment on squash infected with Meloidogyne incognita. J. Nematol. 2004, 36, 290-296.

11. Avato, P.; D'Addabbo, T.; Leonetti, P.; Argentieri, M.P. Nematicidal potential of Brassicaceae. Phytochem. Rev. 2013, 12, 791-802. [CrossRef]

12. Valdes, Y.; Viaene, N.; Moens, M. Effects of yellow mustard amendments on the soil nematode community in a potato field with focus on Globodera rostochiensis. Appl. Soil Ecol. 2012, 59, 39-47. [CrossRef]

13. Grabau, Z.J.; Maung, Z.T.Z.; Noyes, D.C.; Baas, D.G.; Werling, B.P.; Brainard, D.C.; Melakeberhan, H. Effects of cover crops on Pratylenchus penetrans and the nematode community in carrot production. J. Nematol. 2017, 49, 114-123. [CrossRef] [PubMed]

14. D'Addabbo, T.; Avato, P.; Tava, A. Nematicidal potential of materials from Medicago spp. Eur. J. Plant Pathol. 2009, 125, 39-49. [CrossRef]

15. D'Addabbo, T.; Radicci, V.; Avato, P.; Tava, A. Use of pelleted Medicago sativa meal for the control of root-knot and cyst nematodes. Acta Hortic. 2010, 883, 303-308.

16. D'Addabbo, T.; Carbonara, T.; Leonetti, P.; Radicci, V.; Tava, A.; Avato, P. Control of plant parasitic nematodes with active saponins and biomass from Medicago sativa. Phytochem. Rev. 2011, 10, 503-519. [CrossRef]

17. D'Addabbo, T.; Argentieri, M.P.; Żuchowski, J.; Biazzi, E.; Tava, A.; Oleszek, W.; Avato, P. Activity of saponins from Medicago species against phytoparasitic nematodes. Plants 2020, 9, 443. [CrossRef] [PubMed]

18. Argentieri, M.P.; D'Addabbo, T.; Tava, A.; Agostinelli, A.; Jurzysta, M.; Avato, P. Evaluation of nematicidal properties of saponins from Medicago spp. Eur. J. Plant Pathol. 2008, 120, 189-197. [CrossRef]

19. Rodriguez-Kabana, R. Organic and inorganic nitrogen amendments to soil as nematode suppressants. J. Nematol. 1986, 18, 129-134.

20. Janzen, H.H.; McGinn, S.M. Volatile loss of nitrogen during decomposition of legume green manure. Soil Biol. Biochem. 1991, 23, 291-297. [CrossRef]

21. Yeates, G.W.; Bongers, T.D.; De Goede, R.G.M.; Freckman, D.W.; Georgieva, S.S. Feeding habits in soil nematode families and genera-an outline for soil ecologists. J. Nematol. 1993, 25, 315-331.

22. Ekschmitt, K.; Bakonyi, G.; Bongers, M.; Bongers, T.; Boström, S.; Dogan, H.; Harrison, A.; Kallimanis, A.; Nagy, P.; O’Donnell, A.G.; et al. Wolters V Effects of the nematofauna on microbial energy and matter transformation rates in European grassland soils. Plant Soil 1999, 212, 45-61. [CrossRef]

23. Neher, D.A. Role of nematodes in soil health and their use as indicators. J. Nematol. 2001, 33, 161-168. [PubMed]

24. Ferris, H.; Bongers, T.; de Goede, R.G.M. A framework for soil food web diagnostics: Extension of the nematode faunal analysis concept. Appl. Soil Ecol. 2001, 18, 13-29. [CrossRef]

25. Briar, S.S.; Grewal, P.S.; Somasekhar, N.; Stinner, D.; Miller, S.A. Soil nematode community, organic matter, microbial biomass and nitrogen dynamics in field plots transitioning from conventional to organic management. Appl. Soil Ecol. 2007, 37, 256-266. [CrossRef]

26. Cheng, Z.; Grewal, P.S.; Stinner, B.R.; Hurto, K.A.; Hamza, H.B. Effects of long-term turfgrass management practices on soil nematode community and nutrient pools. Appl. Soil Ecol. 2008, 38, 174-184. [CrossRef]

27. Neher, D.A. Ecology of plant and free-living nematodes in natural and agricultural soil. Ann. Rev. Phytopathol. 2010, 48, 371-394. [CrossRef]

28. Bongers, T.; Bongers, M. Functional diversity of nematodes. Appl. Soil Ecol. 1998, 10, 239-251. [CrossRef]

29. Bongers, T.; Ferris, H. Nematode community structure as a bioindicator in environmental monitoring. Trends Ecol. Evol. 1999, 14, 224-228. [CrossRef] 
30. Ferris, H.; Matute, M.M. Structural and functional succession in the nematode fauna of a soil food web. Appl. Soil Ecol. 2003, 23, 93-110. [CrossRef]

31. Bongers, T. The maturity index: An ecological measure of environmental dis-turbance based on nematode species composition. Oecologia 1990, 83, 14-19. [CrossRef]

32. Ferris, H. Form and function: Metabolic footprints of nematodes in the soil food web. Eur. J. Soil Biol. 2010, 46, 97-104. [CrossRef]

33. Urzelai, A.; Hernández, A.J.; Pastor, J. Biotic indices based on soil nematode communities for assessing soil quality in terrestrial ecosystems. Sci. Tot. Envir. 2000, 247, 253-261. [CrossRef]

34. Mulder, C.; Schouten, A.J.; Hund-Rinke, K.; Breure, A.M. The use of nematodes in ecological soil classification and assessment concepts. Ecotoxicol. Environ. Saf. 2005, 62, 278-289. [CrossRef] [PubMed]

35. Sánchez-Moreno, S.; Nicola, N.L.; Ferris, H.; Zalom, F.G. Effects of agricultural management on nematode-mite assemblages: Soil food web indices as predictors of mite community composition. Appl. Soil Ecol. 2009, 41, 107-117. [CrossRef]

36. Bulluck, L.R., III; Barker, K.R.; Ristaino, J.B. Influences of organic and synthetic soil fertility amendments on nematode trophic groups and community dynamics under tomatoes. Appl. Soil Ecol. 2002, 21, 233-250. [CrossRef]

37. McSorley, R.; Frederick, J.J. Nematode population fluctuations during decomposition of specific organic amendments. J. Nematol. 1999, 31, 37-44.

38. Gruver, L.S.; Weil, R.R.; Zasada, I.A.; Sardanelli, S.; Momen, B. Brassicaceous and rye cover crops altered free-living soil nematode community composition. Appl. Soil Ecol. 2010, 45, 1-12. [CrossRef]

39. Chen, J.; Ferris, H. The effects of nematode grazing on nitrogen mineralization during fungal decomposition of organic matter. Soil Biol. Biochem. 1999, 31, 1265-1279. [CrossRef]

40. Renčo, M.; Sasanelli, N.; D’Addabbo, T.; Papajova, I. Soil nematode community changes associated with compost amendments. Nematology 2010, 12, 681-692. [CrossRef]

41. $\mathrm{Hu}, \mathrm{C}$; $\mathrm{Qi}, \mathrm{Y}$. Abundance and diversity of soil nematodes as influenced by different types of organic manure. Helminthologia 2010, 47, 58-66. [CrossRef]

42. Nahar, M.S.; Grewal, P.S.; Miller, S.A.; Stinner, D.; Stinner, B.R.; Kleinhenz, M.D.; Wszelakia, A.; Doohan, D. Differential effects of raw and composted manure on nematode community, and its indicative value for soil microbial, physical and chemical properties. Appl. Soil Ecol. 2006, 34, 140-151. [CrossRef]

43. Tabarant, P.; Villenave, C.; Risède, J.M.; Roger-Estrade, J.; Dorel, M. Effects of organic amendments on plant-parasitic nematode populations, root damage, and banana plant growth. Biol. Fertil. Soils 2011, 47, 341-347. [CrossRef]

44. Treonis, A.M.; Austin, E.E.; Buyer, J.S.; Maul, J.E.; Spicer, L.; Zasada, I.A. Effects of organic amendment and tillage on soil microorganisms and microfauna. Appl. Soil Ecol. 2010, 46, 103-110. [CrossRef]

45. McSorley, R.; Gallaher, R.N. Effect of yard waste compost on nematode densities and maize yield. J. Nematol. 1996, 28, 655-660. [PubMed]

46. Garcia-Alvarez, A.; Arias, M.; Díez-Rojo, M.A.; Bello, A. Effect of agricultural management on soil nematode trophic structure in a Mediterranean cereal system. Appl. Soil Ecol. 2004, 27, 197-210. [CrossRef]

47. Wang, K.H.; McSorley, R.; Marshall, A.; Gallaher, R.N. Influence of organic Crotalaria juncea hay and ammonium nitrate fertilizers on soil nematode communities. Appl. Soil Ecol. 2006, 31, 186-198. [CrossRef]

48. Okada, H.; Harada, H. Effects of tillage and fertilizer on nematode communities in a Japanese soybean field. Appl. Soil Ecol. 2007, 35, 582-598. [CrossRef]

49. Forge, T.A.; Bittman, S.; Kowalenko, C.G. Responses of grassland soil nematodes and protozoa to multi-year and single-year applications of dairy manure slurry and fertilizer. Soil Biol. Biochem. 2005, 37, 1751-1762. [CrossRef]

50. Porazinska, D.L.; Duncan, L.W.; McSorley, R.; Graham, J.H. Nematode communities as indicators of status and processes of a soil ecosystem influenced by agricultural management practices. Appl. Soil Ecol. 1999, 13, 69-86. [CrossRef]

51. DuPont, S.T.; Ferris, H.; Van Horn, M. Effects of cover crop quality and quantity on nematode-based soil food webs and nutrient cycling. Appl. Soil Ecol. 2009, 41, 157-167. [CrossRef]

52. Leroy, B.; Reheul, D.; Moens, M.; Ferris, H.; De Sutter, N. Short-term nematode population dynamics as influenced by the quality of exogenous organic matter. Nematology 2009, 11, 23-38. [CrossRef]

53. Termorshuizen, A.; Korthals, G.; Thoden, T. Organic amendments and their influences on plant-parasitic and free-living nematodes: A promising method for nematode management? Nematology 2011, 13, 133-153. [CrossRef]

54. Ferris, H.; Sánchez-Moreno, S.; Brennan, E.B. Structure, functions and interguild relationships of the soil nematode assemblage in organic vegetable production. Appl. Soil Ecol. 2012, 61, 16-25. [CrossRef]

55. Hooper, D.J.; Hallmann, J.; Subottin, A.S. Methods for Extraction, Processing and Detection of Plant and Soil Nematodes. In Plant Parasitic Nematodes in Subtropical and Tropical Agriculture, 2nd ed.; Luc, M., Sikora, R.A., Bridge, J., Eds.; CABI Publishing: Wallingford, UK, 2005; pp. 53-86.

56. Van Bezooijen, J. Methods and Techniques for Nematology; Wageningen University: Wageningen, The Netherlands, $2006 ;$ p. 112.

57. Brzeski, M.W. Nematodes of Tylenchida in Poland and Temperate Europe; Museum of Institute of Zoology, Polish Academy of Sciences: Warsaw, Poland, 1998; p. 397.

58. Loof, P.A.A. Nematoda: Adenophorea (Dorylaimida); Spektrum Akademischer Verlag: Heidelberg/Berlin, Germany, $1999 ;$ p. 264.

59. Siddiqi, M.R. Tylenchida, Parasites of Plants and Insects, 2nd ed.; CABI Bioscience: Egham, UK, 2000; p. 848. 
60. Geraert, E. The Tylenchidae of the World. Identification of the Family Tylenchidae (Nematoda); Academia Press: Ghent, Belgium, 2008; p. 540.

61. Wasilewska, L. Trophic classification of soil and plant nematodes. Wiadom. Ekol. 1971, 17, 379-388.

62. Sieriebriennikov, B.; Ferris, H.; de Goede, R.G. NINJA: An automated calculation system for nematode-based biological monitoring. Eur. J. Soil Biol. 2014, 61, 90-93. [CrossRef]

63. Yeates, G.W. Modification and qualification of the nematode maturity index. Pedobiologia 1994, 38, 97-101.

64. Andrássy, I. The determination of volume and weight of nematodes. Acta Zool. 1956, 2, 1-15.

65. Finney, D.J. Statistical Method in Biological Assay, 3rd ed.; Charles Griffin \& Company Ltd.: High Wycombe, UK, 1978 ; p. 5. 